\title{
Algorithm for the Fewest Arms Required by Multi- Band Linear Dipole Antenna
}

\author{
Bing Xiao, Hang Wong, Yichen Wei, and Kwan L. Yeung
}

\begin{abstract}
Small linear dipole antennas with a multi-band characteristic are necessary for many strip or bar shaped gateway devices of the Internet of Things (IoT), for the connectivity in various communication protocols. However, the conventional methodology of designing multi-band dipole antennas is generally empirically based. More frequency bands usually mean even more arms/slots, which results in an increasingly bulky antenna. In this paper, we will introduce an algorithm of using the fewest arms to design a multi-band linear dipole antenna. This algorithm is based on sharing arms after the effective ranges of mode excitation are determined by characteristic mode analysis (CMA). By this algorithm, an exemplified designed penta-band dipole antenna is effective in covering $433,868,1176,1575$, and $2450 \mathrm{MHz}$ bands for LPWAN, GNSS, and ISM applications, with only 2.5 pairs of arms. $50 \%$ of arms are reduced in comparison to traditional methods. This algorithm is convenient in practical dipole antenna design, and greatly simplifies the antenna structure so that they could be mounted into small IoT devices.
\end{abstract}

Index Terms-GPS, Internet of Things (IoT), LPWAN, smart glasses, smart pencil, smart wristband, theory of characteristic mode (TCM), wireless smart device

\section{INTRODUCTION}

$\mathrm{M}$ any of the objects surrounding us are being connected to the Internet of Things (IoT) at present and in the future [1], [2]. There are various IoT communication protocols. Thus, IoT peripheral nodes usually communicate on different frequencies. For example, the low-power wide-area network (LPWAN) is a type of IoT network designed for long-range wide-area wireless communications at a low bit rate and low power consumption. It comprehends multiple standards, such as Lora [3], Zigbee [4], Sigfox [5], NB-IoT [6], etc. Lora, Zigbee, and Sigfox use license-free ISM bands, such as 433 $\mathrm{MHz}, 868 \mathrm{MHz}$, and $2450 \mathrm{MHz}$. While NB-IoT uses licensed narrow bands owned by mobile network operators. The selection of their operating frequency will consider transmission distance, power consumption, local regulations, cost control, etc. Apart from LPWAN, Bluetooth Low Energy

This work was supported in part by the Research Grants Council of the Hong Kong SAR, China with a TRS project (Project No. T42-103/16-N), a CRF project (Project No. CityU C1020-19E), and the CityU Strategic Research Grant (Project No. SRG-Fd 7005227).

Bing Xiao and L. K. Yeung are with the Department of Electrical and Electronic Engineering, The University of Hong Kong, Hong Kong.

H. Wong is with The State Key Laboratory of Terahertz and Millimeter Waves, and Shenzhen Research Institute, Department of Electrical Engineering, City University of Hong Kong, Hong Kong (e-mail: hang.wong@cityu.edu.hk).

Yichen Wei is with the Department of Computer Science, The University of Hong Kong, Hong Kong.
(BLE) and Wireless Local Area Network (WLAN) operate at $2450 \mathrm{MHz}$.

On the other hand, an IoT gateway is a network node that connects the above-mentioned multiple different networks [7]. Some wireless smart devices, such as wireless routers, smart wristbands, smart pencils, and smart glasses, are all potential IoT gateways in the future. Moreover, when the IoT gateways directly communicate with various IoT peripherals, the gateway device could also serve as an edge server, greatly facilitating edge computing for smart home and city [8].

Furthermore, the global navigation satellite system (GNSS) is an essential function in assisting some mobile IoT gateway devices in positioning. For example, smart glasses can improve their function of augmented reality (AR) by GNSS. Recently, in addition to the commonly used global positioning system (GPS) L1 band, GPS L5 band has also been adopted massively by commercial wireless products in order to enhance the accuracy of positioning [9].

However, in the whole wireless communication system in IoT devices, antenna design is a prominent difficulty. Many of IoT devices are small in size. But the length of the antenna is directly inversely proportional to the operating frequency. Thus, its size is difficult to be compressed. On the other hand, the antennas become even bulky when required to cover multiple frequency bands, even making the antenna could not be mounted on many strip or bar shaped devices, such as smart wristbands, smart pencils, and smart glasses.

Many small antennas in mobile devices, such as Planar Inverted-F Antennas (PIFAs), Inverted-F Antennas (IFAs), monopole antennas, and patch antennas [10], require a large-size ground, which many small IoT devices do not possess. In this condition, multi-band dipole antennas are more suitable.

Present research on multi-band dipole antennas are mostly concentrated on dual or triple frequency bands, as summarized and compared in Table I.

Adding several pairs of arms/slots is the most common method for introducing additional frequency bands for dipole antennas. Some of them were achieved by directly connecting additional arms to the primary pair of arms. In [11], a shorter pair of arms was connected to the dipole antenna for a higher frequency band. In [12], two more shorter meandered pairs of arms were connected to the primary arms to make it a tripleband dipole antenna. In [13], three connected pairs of slots fed by a coplanar waveguide (CPW) were designed for a tripleband antenna.

Meanwhile, some of the multi-arm/slot dipole antennas were achieved by coupling. In [14], [15], two coupled arms were placed near the primary arms for a tri-band dipole antenna. In [16], a dual-band and a tri-band dipole antennas were proposed by coupling one or two parasitic half-wavelength strips above 
the primary arms for the higher frequency bands. In [17], a pair of coupled arms were placed near the dipole, extending the dipole's length for an additional lower frequency band. This antenna adopted one-wavelength radiation for both two frequencies. Moreover, slots could be embedded in a dipole for an additional band [18].

For this method of adding pairs of arms/slots, the radiation patterns of the multiple resonant frequencies could keep omnidirectional. However, the ratio between the pairs of arms/slots and the required frequency bands is at least $1: 1$. When one more frequency band is required, at least one more pair of arms is needed.

Higher-order harmonic modes of the dipole antenna could also be considered for more frequency bands. When the feeding point is at exactly the middle of the dipole, the odd-order modes could be excited. In [19], the dipole antenna excited the fundamental and the third-order mode, i.e., the half-wavelength $(0.5 \lambda)$ mode and the one-and-a-half wavelength $(1.5 \lambda)$ mode. Some slots are embedded in the dipole to tune the higher-order mode. However, these antennas by virtue of harmonic modes have a disadvantage that the higher-order harmonic frequencies should be close to the multiples of the fundamental frequency. Besides, it is quite difficult to manipulate multiple resonant frequencies at the same time.

For a more intuitive comparison, Fig. 1 exhibits the number of pairs of arms/slots $N_{\mathrm{a}}$ vs. that of the frequency bands $N_{\mathrm{f}}$ for multi-band dipole antennas. The above-mentioned dipole antennas are mostly located on or above the line of $N_{\mathrm{a}}=N_{\mathrm{f}}$, especially when more than two frequency bands are required. Thus, the antenna structure becomes increasingly bulky with more arms when more frequency bands are required. However, IoT devices are usually small in size. A new path of thought for designing multi-band dipole antennas is required to compress the antenna size to fit the requirement of IoT devices.

In this paper, firstly, the conditions of exciting any specific mode of a dipole antenna are analyzed by the theory of characteristic mode (TCM). Then we know that each mode could be excited in ranges rather than just at a single location. In this condition, we could make some arms shared by multiple modes. Then, based on that, an algorithm is designed to calculate the fewest arms for any linear multi-band dipole antenna. When the desired frequencies are the input, the lengths of the fewest dipole's arms are the output. The algorithm could compress the size of the multi-band dipole antenna to the minimum. Antennas designed by this algorithm could achieve multi-band radiation while conforming to various narrow surfaces, as shown in Fig. 2. They could be mounted on various strip/bar-shaped IoT devices, such as wireless routers, smart pencils, smart wristbands, and smart glasses, as shown in Fig. 3.

In the example presented for illustrating the algorithm, a dipole antenna is calculated and designed by this algorithm to cover 5 commonly used frequency bands (ISM bands of 433 $\mathrm{MHz}, 868 \mathrm{MHz}$, and $2450 \mathrm{MHz}$; GPS L5 of $1176 \mathrm{MHz}$ and L1 of $1575 \mathrm{MHz}$ ) with only 2.5 pairs of arms. $50 \%$ of arms are reduced in comparison to the traditional method.
TABLE I

MULTI-BAND DIPOLE ANTENNAS

\begin{tabular}{|c|c|c|c|}
\hline & $\begin{array}{c}\text { Frequency } \\
\text { bands }\left(N_{\mathrm{f}}\right) \uparrow\end{array}$ & $\begin{array}{c}\text { Pairs of } \\
\text { arms/slots }\left(N_{\mathrm{a}}\right)\end{array}$ & Methods \\
\hline$[18]$ & 2 & 3 & Connecting slots \\
\hline [11] & 2 & 2 & Connecting arms \\
\hline [17] & 2 & 2 & Coupling arms \\
\hline$[16]^{*}$ & 2 & 2 & Connecting arms \\
\hline [19] & 2 & 1 & Harmonics \\
\hline [20] & 3 & 4 & Connecting arms \\
\hline [13] & 3 & 3 & Connecting slots \\
\hline$[16]^{* *}$ & 3 & 3 & Connecting arms \\
\hline [12] & 3 & 3 & Connecting arms \\
\hline$[14]$ & 3 & 3 & Coupling arms \\
\hline [15] & 3 & 3 & Coupling arms \\
\hline [21] & 3 & 3 & Connecting arms \\
\hline [22] & 5 & 5 & Connecting slots \\
\hline Proposed & 5 & 2.5 & Sharing arms \\
\hline
\end{tabular}

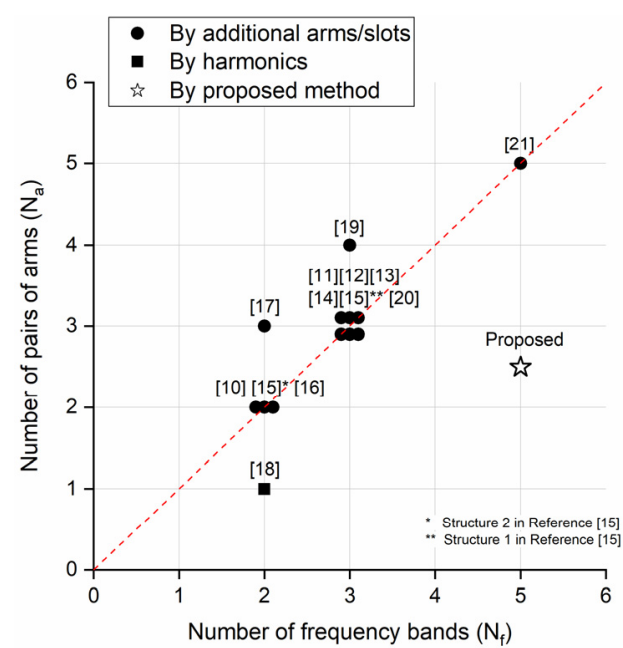

Fig. 1. Number of pairs of arms $N_{\mathrm{a}}$ vs. number of frequency bands $N_{\mathrm{f}}$ for various multi-band dipole antennas

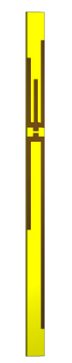

(a)

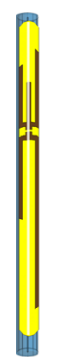

(b)

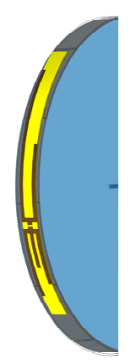

(c)
Fig. 2. The antenna conforms to narrow (a) flat plane, (b) latitude curved surface, and (c) longitude curved surface
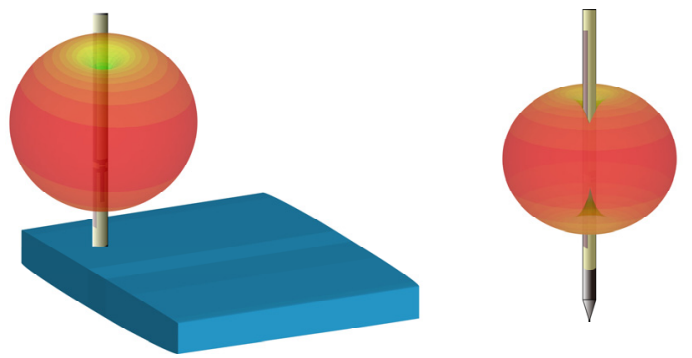


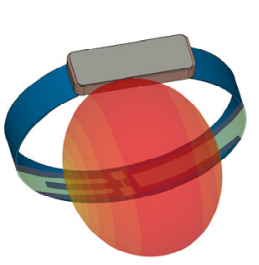

(c) (b)

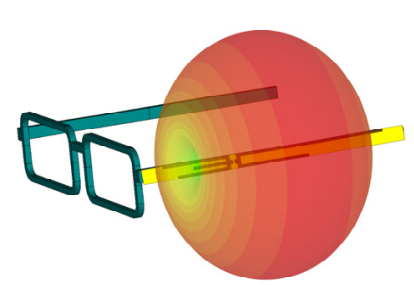

(d)
Fig. 3. Radiation performance (at $433 \mathrm{MHz}$ ) of the antenna when mounted on (a) wireless router, (b) smart pencil, (c) smart wristband, and (d) smart glasses

\section{Characteristic Mode Analysis of Feeding Location}

In order to calculate the effective ranges of excitation for each mode of a dipole antenna, we introduce the theory of characteristic mode (TCM). TCM was first proposed by Garbacz in 1968 [23] and then refined by Harrington and Mautz in 1971 [24]. It could provide the characteristic modes of an arbitrarily-shaped metallic structure, without considering the feeding arrangement. It brings physical insight into the radiating phenomena of the antenna. The characteristic modes are obtained by solving an eigenvalue equation derived from the method of moments (MoM): $\mathrm{X}\left(\mathbf{J}_{\mathrm{n}}\right)=\Lambda_{\mathrm{n}} \mathrm{R}\left(\mathbf{J}_{\mathrm{n}}\right)$, where $X$ and $R$ are the imaginary and real parts of an impedance matrix $Z$. $\mathbf{J}_{\mathrm{n}}$ is the eigencurrent of the $n$-th mode. At a given frequency, we can determine whether or not the characteristic mode is at resonance by looking at its eigenvalue $\Lambda_{\mathrm{n}}$. When $\Lambda_{\mathrm{n}}$ is near 0 , the mode is at resonance. The real current $\mathbf{J}$ on a conductive surface $S$ can be expressed as a linear superposition of the normalized eigencurrents $\mathbf{J}=\sum \alpha_{\mathrm{n}} \mathbf{J}_{\mathrm{n}}$. Where $\alpha_{\mathrm{n}}$ is the modal weighting coefficient (MWC) of the $n$-th characteristic mode, indicating how strongly the mode $\mathbf{J}_{\mathrm{n}}$ is excited. $\alpha_{\mathrm{n}}$ can be expressed as:

$$
\alpha_{n}=\frac{\left\langle\mathbf{J}_{n}, \mathbf{E}^{i}\right\rangle}{1+j \Lambda_{n}}=\frac{1}{1+j \Lambda_{n}} \cdot \oiint_{S} \mathbf{J}_{n} \cdot \mathbf{E}^{i} d S
$$

$\mathbf{E}^{\mathrm{i}}$ is the impressed electric field on the conductive surface $S$. Thus, $\left\langle\mathbf{J}_{\mathrm{n}}, \mathbf{E}^{\mathrm{i}}\right\rangle$ is actually the modal excitation coefficient (MEC) of Mode $n$, indicating the coupling degree between the intrinsic mode and the external incident field from the excitation source. From this expression, we can see that a mode can be successfully excited when it has a near-zero $\Lambda_{\mathrm{n}}$ and an excitation source imposed with a high MEC at the desired frequency. However, MWC varies with the feeding location for each mode of a dipole antenna.

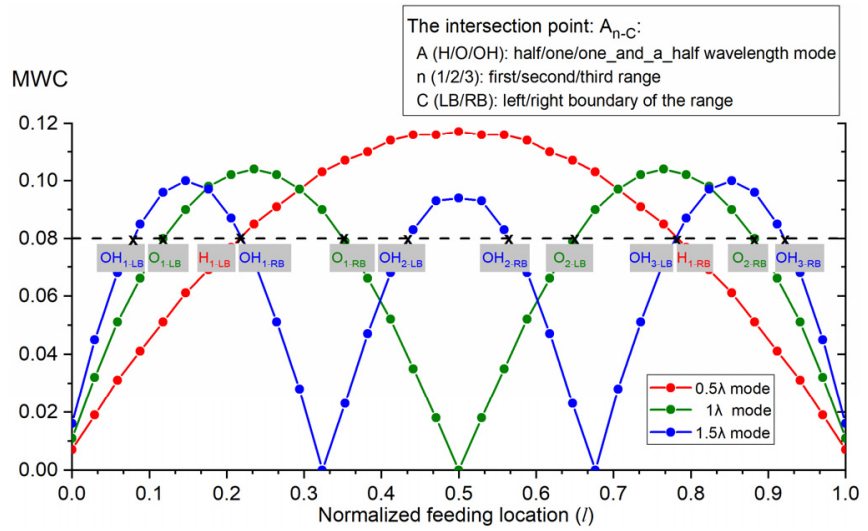

Fig. 4. MWC vs. feeding location for $0.5 \lambda, 1 \lambda$, and $1.5 \lambda$ modes

For a perfectly conducting narrow strip (thus the transverse modes could be neglected in the concerned frequencies), MWCs vs. the feeding locations for $0.5 \lambda, 1 \lambda$, and $1.5 \lambda$ modes are depicted in Fig. 4, calculated by the characteristic mode analysis module of FEKO [25]. The fourth and even higherorder modes could also be calculated by this method. The Xaxis shows the normalized feeding location, while the $\mathrm{Y}$-axis shows the MWCs. If we wish to excite only one mode, then we should excite at the location where the mode has the highest MWC, corresponding to the lowest reflection coefficient. However, if we wish to excite more than one mode simultaneously with a single feeding port, we should balance the MWCs for all modes. For example, when MWC $>0.08$ is set as the criterion that the mode is excited, the intersection points of MWC curves and the line of $\mathrm{MWC}=0.08$ are annotated as in Fig. 4. We can see that the commonly used $0.5 \lambda$ mode can be easily excited in a wide range in the middle part of the strip $\left(\mathrm{H}_{1-\mathrm{LB}}<l<\mathrm{H}_{1-\mathrm{RB}}\right)$. The $1 \lambda$ mode can be excited in two separate ranges in the left and the right $\left(\mathrm{O}_{1-\mathrm{LB}}<l<\mathrm{O}_{1-\mathrm{RB}}\right.$ or $\mathrm{O}_{2-}$ $\left.{ }_{\mathrm{LB}}<l<\mathrm{O}_{2-\mathrm{RB}}\right)$. The $1.5 \lambda$ mode can be excited in three separate ranges in the left, middle, and right $\left(\mathrm{OH}_{1-\mathrm{LB}}<l<\mathrm{OH}_{1-\mathrm{RB}}\right.$ or $\mathrm{OH}_{2-}$ LB $<l<\mathrm{OH}_{2-\mathrm{RB}}$ or $\left.\mathrm{OH}_{3-\mathrm{LB}}<l<\mathrm{OH}_{3-\mathrm{RB}}\right)$. For example, if the feeding port is located at $l=0.3$, the $0.5 \lambda$ mode and $1 \lambda$ mode could be excited because their MWC $>0.08$, while the $1.5 \lambda$ mode fails to be excited because its MWC is much smaller than 0.08 .

Here, $L$ and $R$ will represent the lengths of the left arm and right arm in a pair. The ratio of them, $L / R$ is calculated particularly for the relative feeding location for the next-step algorithm. If the $0.5 \lambda$ mode can be excited, then $L / R$ should be in:

$$
I_{1}: \quad \frac{H_{1 \_L B}}{1-H_{1 \_L B}}<\frac{L}{R}<\frac{H_{1_{-} R B}}{1-H_{1 \_R B}}
$$

If the $1 \lambda$ mode can be excited, $L / R$ should be in:

$$
I_{2}: \quad \begin{aligned}
\frac{O_{1 \_} L B}{1-O_{1_{-} L B}} & <\frac{L}{R}<\frac{O_{1_{-} R B}}{1-O_{1_{-} R B}} \\
\text { or } \quad \frac{O_{2_{-} L B}}{1-O_{2_{-} L B}}<\frac{L}{R} & <\frac{O_{2_{-} R B}}{1-O_{2_{-} R B}}
\end{aligned}
$$

If the $1.5 \lambda$ mode is excited, then $L / R$ should be in: 


$$
\begin{aligned}
I_{3}: \quad \frac{O H_{1_{-} L B}}{1-\mathrm{OH}_{1_{-} L B}}<\frac{L}{R}<\frac{O H_{1_{-} R B}}{1-\mathrm{OH}_{1_{-} R B}} \\
\quad \text { or } \frac{O H_{2_{-} L B}}{1-\mathrm{OH}_{2} L B}<\frac{L}{R}<\frac{O H_{2_{-} R B}}{1-\mathrm{OH}_{2_{-} R B}} \\
\text { or } \frac{O H_{3_{-} L B}}{1-\mathrm{OH}_{3_{-} L B}}<\frac{L}{R}<\frac{O H_{3_{-} R B}}{1-\mathrm{OH}_{3_{-} R B}}
\end{aligned}
$$

By the characteristic mode analysis, we know for a pair of arms, the effective feeding location could be in ranges instead of a single point. By taking advantage of this feature, different modes can share some arms, resulting in a reduction of the number of arms for a multi-band dipole antenna.

\section{AlgORITHM FOR CALCULATING LENGTHS OF ARMS}

The flow diagram of the algorithm is shown in Fig. 5. The whole algorithm is conducted by searching for sets of lengths that satisfy the conditions of exciting the desired multiple resonances step by step from lower to higher frequencies. A set of lengths of the dipole's arms is defined as $\left[L_{1}, L_{2}, \ldots, L_{\mathrm{u}}, R_{1}\right.$, $R_{2}, \ldots, R_{\mathrm{v}}$ ], representing the antenna has $u$ left arms and $v$ right arms. $f_{1}$ to $f_{\mathrm{t}}$ denote the center-frequencies of the required $t$ frequency bands, while $\lambda_{1}$ to $\lambda_{\mathrm{t}}$ are the corresponding guided wavelengths on the selected substrate of the frequencies $f_{1}$ to $f_{\mathrm{t}}$. $\lambda_{\mathrm{i}}=c /\left(f_{i} \times \sqrt{\varepsilon_{\mathrm{re}}}\right)$, where $c$ is the light speed in vacuum and $\varepsilon_{\mathrm{re}}$ is the effective relative permittivity. There are various methods to calculate the effective permittivity when an antenna is placed on a specific substrate without a ground plane, such as $\left(\varepsilon_{\mathrm{r}}+1\right) / 2$, which is suitable for a nearly semi-infinite substrate, closed- form expression which is based on conformal mapping [26]. However, we find that they are not universal in precisely calculating the effective permittivity. Here we adopt the method in [27] by comparing the resonant frequencies of a dipole in free space and the same dipole printed on the examined substrate by 3D simulation software. Then the ratio between them will be the square root of the effective relative permittivity $\varepsilon_{\text {re }}$. Here, for a $412 \mathrm{~mm} \times 20 \mathrm{~mm} \mathrm{0.8-mm-thick} \mathrm{RO4003C}\left(\varepsilon_{\mathrm{r}}=3.38\right)$ substrate with the dipole arm width $W=4 \mathrm{~mm}$ and feed gap $d$ $=2 \mathrm{~mm}$, the effective relative permittivity $\varepsilon_{\mathrm{re}}=1.87$.

$I_{1}$ to $I_{\mathrm{p}}$ are the ranges of $L / R$ for effective excitation of Mode $1(1 \times 0.5 \lambda$ mode $)$ to Mode $p(p \times 0.5 \lambda$ mode $)$, including the above-mentioned (2)-(4). $L_{\mathrm{m}}$ denotes $L_{1}$, or $L_{2}$, or $L_{3} \ldots$, while $R_{\mathrm{n}}$ denotes $R_{1}$ or $R_{2}$, or $R_{3} \ldots$. For each resonance, the longer the current path $\left(L_{\mathrm{m}}+R_{\mathrm{n}}\right)$, the lower the resonant frequency.

As shown in Fig. 5, in the beginning, a set of $\left[L_{1}, L_{2}, \ldots, L_{\mathrm{u}}\right.$, $\left.R_{1}, R_{2}, \ldots, R_{\mathrm{v}}\right]$ is evaluated. Firstly, we will see whether there exists a combination of $L_{\mathrm{m}}$ and $R_{\mathrm{n}}$ that can satisfy the requirements of exciting $f_{1}$ : both the condition of the total length $L_{\mathrm{m}}+R_{\mathrm{n}}$ and the condition of the relative location $L_{\mathrm{m}} / R_{\mathrm{n}}$. If both of them can be satisfied, $0.5 \lambda_{1}$ with $I_{1}$, or $1 \lambda_{1}$ with $I_{2}$, or $1.5 \lambda_{1}$ with $I_{3} \ldots$, or $(p \times 0.5) \lambda_{1}$ with $I_{\mathrm{p}}$, we can go forward to the next module, evaluating the requirement of $f_{2}$. In the module of $f_{2}$, it is possible that $m^{\prime}=m$ and $n^{\prime}=n$, for the condition that one pair of arms could cover both $f_{1}$ and $f_{2}$. More possibly, either $m^{\prime}=m$ or $n^{\prime}=n$, for the condition of one shared arm. Also possibly, $m^{\prime}$ $\neq m$ and $n^{\prime} \neq n$, for the condition of no shared arms. If the requirements of $f_{2}$ module could be satisfied, then it proceeds to the modules of $f_{3}, f_{4}, \ldots, f_{\mathrm{t}}$ successively. For the $f_{\mathrm{t}}$ module, $L_{\mathrm{m}}{ }^{(t-}$ ${ }^{1)}$ and $R_{\mathrm{n}}{ }^{(t-1)}$ should satisfy the conditions of $0.5 \lambda_{\mathrm{t}}$ with $I_{1}$, or $1 \lambda_{\mathrm{t}}$ with $I_{2}$, or $1.5 \lambda_{\mathrm{t}}$ with $I_{3} \ldots$, or $(p \times 0.5) \lambda_{\mathrm{t}}$ with $I_{\mathrm{p}}$.

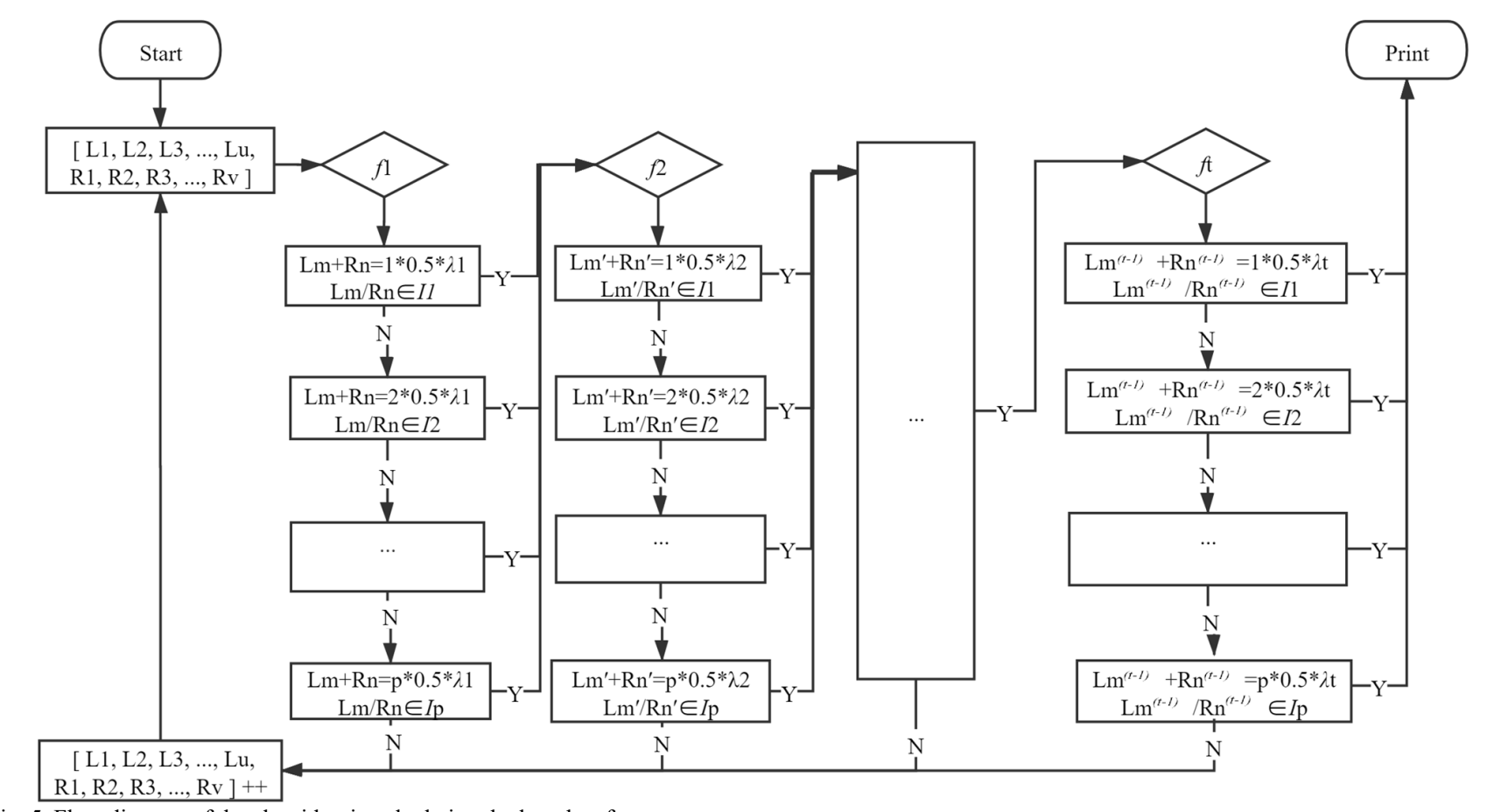

Fig. 5. Flow diagram of the algorithm in calculating the lengths of arms 


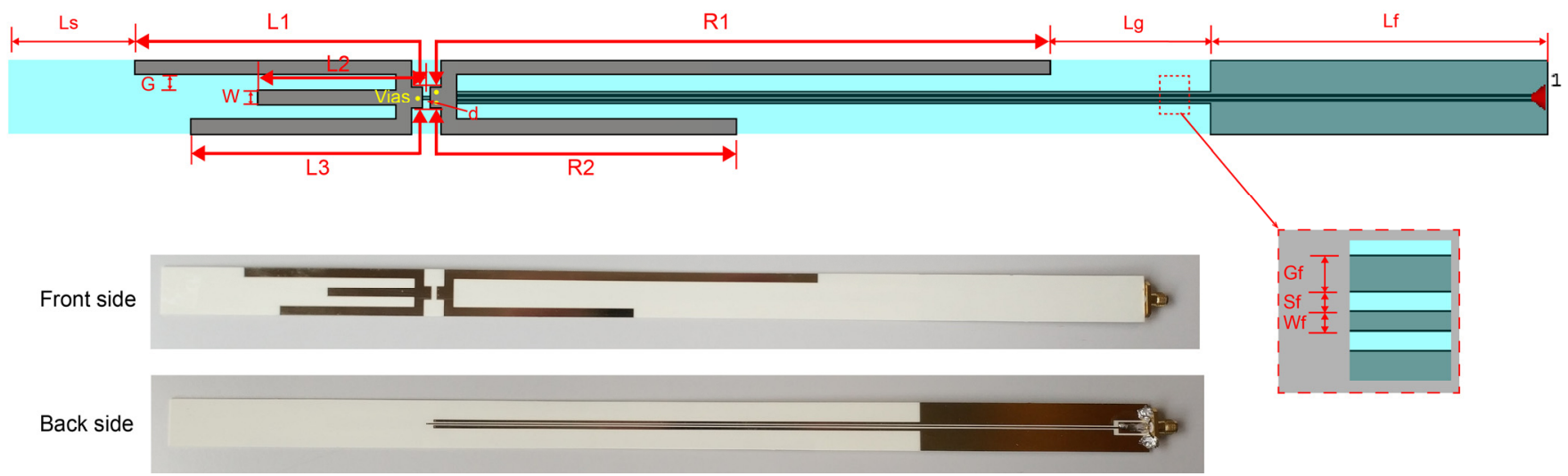

Fig. 6. Schematic and prototype of the exemplified penta-band dipole antenna

If all the $t$ modules are satisfied by this set of values, we can print it out. Then it could be the initial values for the next-step $3 \mathrm{D}$ electromagnetic simulation. If any module cannot be satisfied in this flow path, another set of $\left[L_{1}, L_{2}, \ldots, L_{\mathrm{u}}, R_{1}, R_{2}\right.$, $\left.\ldots, R_{\mathrm{v}}\right]$ will be reevaluated from the beginning. The precisions of $L_{\mathrm{m}}, R_{\mathrm{n}}$, and $\lambda$ can be restricted to $1 \mathrm{~mm}$ or others in a balance of the computing time and the required precision. Then several sets of lengths of arms can be obtained by this algorithm. If the program cannot work out any solutions, then we should increase $u$, or $v$, or both, until it can work out solutions. By this whole process, we could calculate the fewest arms for any multi-band dipole antenna.

From the above algorithm, we could know that the multiband single-pair dipole antennas achieved by harmonics, such as [19], are special cases when we set $u=v=1$. It will have solutions when the higher frequencies are multiples of the fundamental frequency. $t$-band $t$-pair dipole antennas, such as [11], are special cases when we set $p=1$, meaning that only $0.5 \lambda$ modes are utilized. It is applicable to the scenario that the radiation omni-directivity is highly required.

It is noteworthy that for a balance of the operating efficiency and the result validity of the algorithm, some secondary factors are not involved in it. Instead, their effects will be evaluated in the later 3D electromagnetic simulation after the initial values of several sets of values of lengths are acquired. Firstly, at any desired frequency, the surface current of the antenna will be mainly concentrated on the selected pair of arms. At this time, all other arms become shunt stubs, introducing an additional admittance at the feeding port. Secondly, the mutual coupling between adjacent arms is also not considered in the algorithm. Both the factors may cause minor deviations of the final results, such as small frequency deviations and higher reflection coefficient. Thus, the results from the algorithm need further fine-tuning in 3D electromagnetic simulation. For example, lengthen or shorten the arm pair to decrease or increase the resonant frequency, and adjust $L / R$ of a pair to change the input impedance for a better matching.

\section{EXEMPLIFIED CALCUlation AND DESIGN OF A PENTA- BAND DIPOLE ANTENNA}

The algorithm is realized by Python 3.8 [28]. For illustrating and verifying the algorithm, a penta-band dipole antenna will be calculated and designed. $\left[f_{1}, f_{2}, f_{3}, f_{4}, f_{5}\right]$ is $[433,868,1176$, $1575,2450](\mathrm{MHz})$. Thus $t=5$. We limit the antenna to use only the first three modes, thus $p=3$. Then, by the calculation of the algorithm, at least 2.5 pairs (5) of arms are required: $u=3$ and $v=2$. Several sets of results and the corresponding simulated reflection coefficient are shown in Fig. 7. In some solutions, there exist some small frequency deviations and insufficient excitation. The reasons are as analyzed in the previous section. Further fine-tuning with 3D electromagnetic simulation is necessary. Here, the 3rd solution is selected as the initial values.

Thus, this dipole antenna is formed by five arms with these different lengths, forming an octopus shape as shown in Fig. 6. For the convenience of measurement, a coplanar waveguide (CPW) balun is designed to feed the antenna. As shown in Fig. 6 , the feeding structure is on the back side of the substrate, shown in a darker color. At the left end of the feed line, the median strip is connected with the left arms by a via, i.e., a plated-through hole (PTH), while the two outer ground lines are connected with the right arms by two PTHs. However, it is noteworthy that this bulky feeding structure is not necessary for practical IoT devices. The transceiver chip could be mounted near the location between the left and right arms. Finally, the dimensions of the proposed antenna are shown in Fig. 6 and Table III.

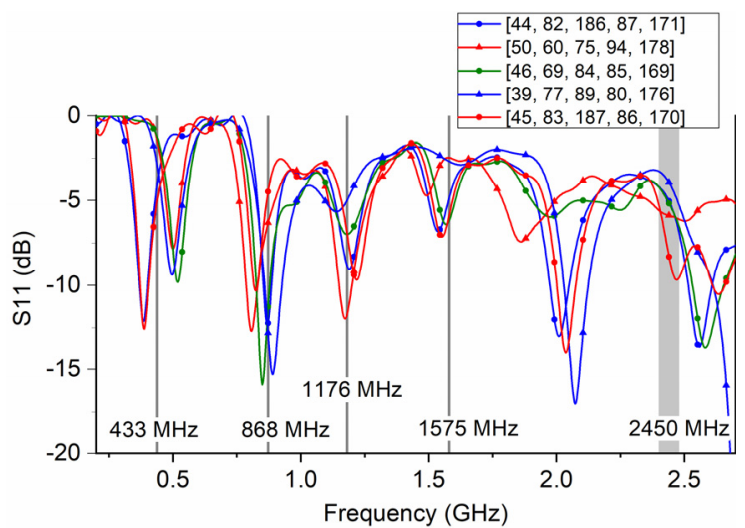

Fig. 7. Simulated reflection coefficient of some solutions of $\left[L_{1}, L_{2}, L_{3}, R_{1}, R_{2}\right]$ worked out by the algorithm 
TABLE III

DIMENSIONS OF THE PROPOSED ANTENNA (Unit: $\mathrm{mm}$ )

\begin{tabular}{cccccccc}
\hline $\boldsymbol{L 1}$ & $\boldsymbol{L} \boldsymbol{2}$ & $\boldsymbol{L} \boldsymbol{3}$ & $\boldsymbol{R} \boldsymbol{1}$ & $\boldsymbol{R} \boldsymbol{2}$ & $\boldsymbol{W}$ & $\boldsymbol{G}$ & $\boldsymbol{d}$ \\
\hline 82 & 44 & 67 & 171 & 87 & 4 & 4 & 2 \\
\hline $\boldsymbol{G}$ & $\boldsymbol{L s}$ & $\boldsymbol{L g}$ & $\boldsymbol{L f}$ & $\boldsymbol{W f}$ & $\boldsymbol{S f}$ & $\boldsymbol{G f}$ & \\
\hline 4 & 34 & 43 & 90 & 0.5 & 0.5 & 1 & \\
\hline
\end{tabular}

(a) $433 \mathrm{MHz}$

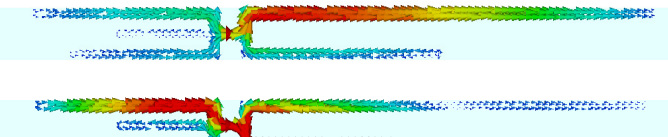

(b) $868 \mathrm{MHz}$

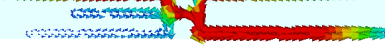

(c) $1176 \mathrm{MHz}$

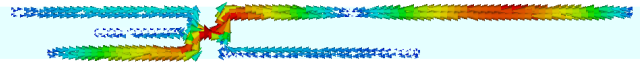

(d) $1575 \mathrm{MHz}$

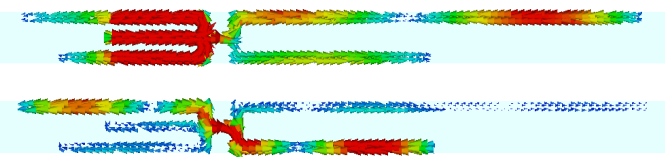

(e) $2450 \mathrm{MHz}$

Fig. 8. Simulated normalized current distribution of the five desired frequencies

Fig. 8 shows the simulated surface current distribution at the five desired frequency bands. We can see that $433 \mathrm{MHz}$ and $868 \mathrm{MHz}$ bands are operating in $0.5 \lambda$ mode. $1176 \mathrm{MHz}$ band is operating in $1 \lambda$ mode. $1575 \mathrm{MHz}$ and $2450 \mathrm{MHz}$ bands are operating in $1.5 \lambda$ band. As expected, some arms are shared by multiple modes. $L_{1}$ is shared by $433 \mathrm{MHz}, 868 \mathrm{MHz}$, and 2450 $\mathrm{MHz} ; L_{2}$ is exclusively used by $1575 \mathrm{MHz} ; L_{3}$ is exclusively used by $1176 \mathrm{MHz} ; R_{1}$ is shared by $433 \mathrm{MHz}, 1176 \mathrm{MHz}$, and $1575 \mathrm{MHz} ; R_{2}$ is shared by $868 \mathrm{MHz}$ and $2450 \mathrm{MHz}$. By this sharing, the number of arms of this penta-band dipole antenna could be reduced to 2.5 pairs.

Moreover, we know in antenna theory, the antenna can be more broadband by occupying a larger volume [29]. For IoT applications, the required bandwidths are usually very narrow because of their property of low-bit-rate transmission, so the arms of the dipole antenna could be very narrow. For other multi-band applications, we could increase the widths of arms according to the requirement of antenna bandwidth.

A prototype, as shown in Fig. 6, is fabricated and then measured by the vector network analyzer (VNA) and the microwave anechoic chamber. The simulated and measured reflection coefficients are shown in Fig. 9. The measured VSWR is smaller than 2.1 in the desired frequency bands. Here, the antenna covers the frequency bands of $433 \mathrm{MHz}(433.05$ $\mathrm{MHz}-434.79 \mathrm{MHz}), 868 \mathrm{MHz}(863 \mathrm{MHz}-870 \mathrm{MHz}), 1176$ $\mathrm{MHz}(1176.45 \mathrm{MHz} \pm 1.023 \mathrm{MHz}), 1575 \mathrm{MHz}(1575.42 \mathrm{MHz}$ $\pm 1.023 \mathrm{MHz})$, and $2450 \mathrm{MHz}(2400 \mathrm{MHz}-2480 \mathrm{MHz})$.

The maximum gain and total efficiency of the antenna are shown in Fig. 10. The simulated and measured total efficiency is higher than $56 \%$ in the desired frequency bands. Then the simulated and measured radiation patterns for the desired frequency bands are shown in Fig. 11. (Due to the limitation of the frequency range of our microwave chamber, we only present the simulated results for the $433 \mathrm{MHz}$ band.) The radiation patterns at the desired frequencies are all similar to those of single-pair dipole antennas. It is noteworthy that there are small deflections from the axial direction for the radiation patterns, when the contributing pair of left and right arms of this mode are not in a line, such as the four higher frequency bands.

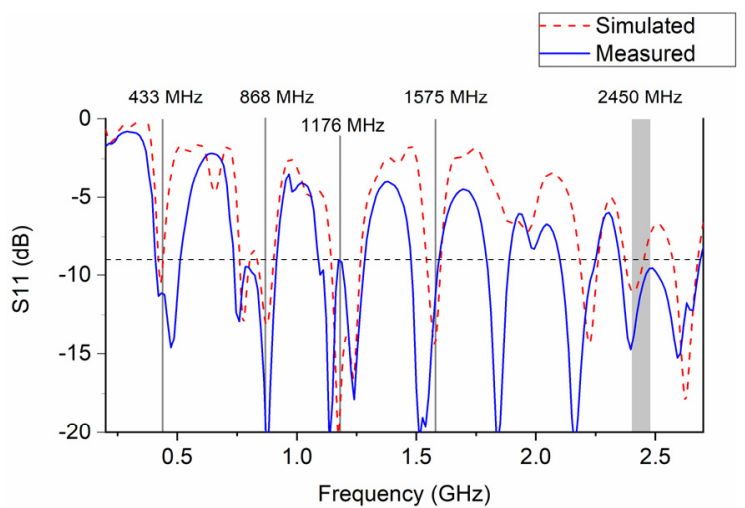

Fig. 9. The simulated and measured reflection coefficient of the proposed antenna

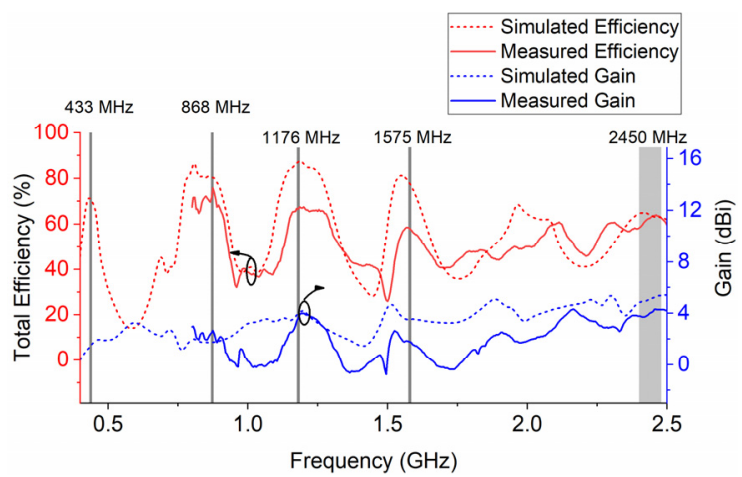

Fig. 10. Simulated and measured total efficiency and the maximum gain vs. frequency of the proposed antenna

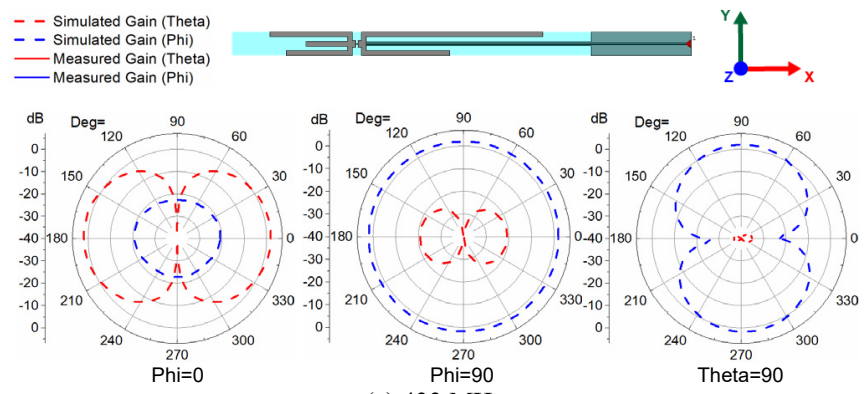

(a) $433 \mathrm{MHz}$

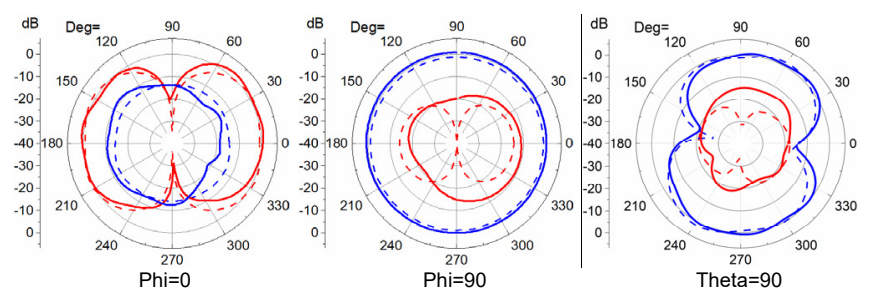

(b) $868 \mathrm{MHz}$ 

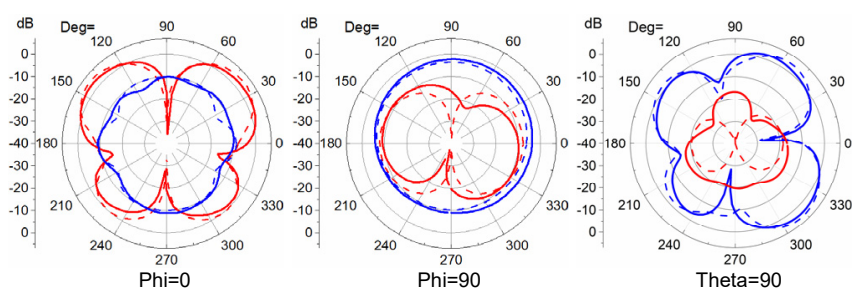

(c) $1176 \mathrm{MHz}$
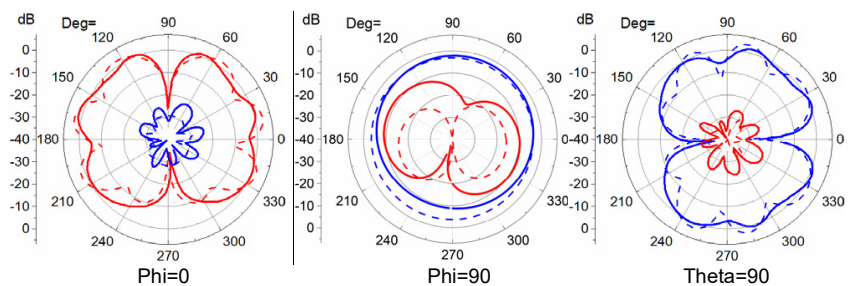

(d) $1575 \mathrm{MHz}$
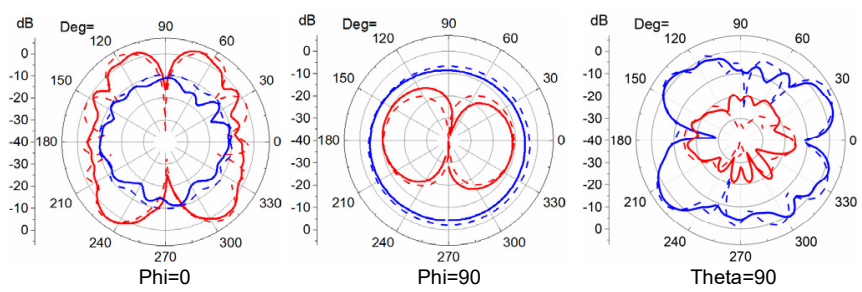

(e) $2450 \mathrm{MHz}$

Fig. 11. Simulated and measured radiation patterns for the desired frequency bands

\section{CONCLUSION}

In this paper, we introduced an algorithm for calculating the fewest arms required by a linear multi-band dipole antenna. The calculated lengths of the arms by the algorithm could serve as the initial values. Then after some fine tuning, the antenna could cover the desired multiple narrow bands. The exemplified penta-band dipole antenna could cover 433, 868, 1176, 1575, and $2450 \mathrm{MHz}$ bands with only 2.5 pairs of arms, facilitating the LPWAN, GNSS, and ISM communications for IoT gateway devices. This algorithm is easy to use and facilitates the rapid and systematic design of linear multi-band dipole antennas for IoT and other applications.

\section{Acknowledgment}

The authors would like to thank Mr. Guang-Hua Sun, Dr. Bo Wang, Dr. Quan-Wei Lin, and Miss Zhuo-Qiao Ji, for their help in measurement.

\section{REFERENCE}

[1] J. Gubbi, R. Buyya, S. Marusic, and M. Palaniswami, "Internet of Things (IoT): A vision, architectural elements, and future directions," Future generation computer systems, vol. 29, no. 7, pp. 1645-1660, 2013.

[2] A. Zanella, N. Bui, A. Castellani, L. Vangelista, and M. Zorzi, "Internet of things for smart cities," IEEE Internet of Things journal, vol. 1, no. 1, pp. 22-32, 2014.

[3] N. Sornin, M. Luis, T. Eirich, T. Kramp, and O. Hersent, "LoRaWAN specification v1. 0," Technical report, LoRa Alliance, Tech. Rep., 2015.

[4] "Zigbee Technical Specifications," Zigbee Alliance. https://zigbeealliance.org/solution/zigbee/ (accessed Jan. 29, 2020).
[5] "Sigfox Device Radio Specifications | Sigfox build" https://build.sigfox.com/sigfox-device-radio-specifications\# (accessed Jan. 29, 2020).

[6] Y. Miao, W. Li, D. Tian, M. S. Hossain, and M. F. Alhamid, "Narrowband internet of things: Simulation and modeling," IEEE Internet of Things Journal, vol. 5, no. 4, pp. 2304-2314, 2017.

[7] T. Zachariah, N. Klugman, B. Campbell, J. Adkins, N. Jackson, and P. Dutta, "The internet of things has a gateway problem," in Proceedings of the 16th international workshop on mobile computing systems and applications, 2015, pp. 27-32.

[8] W. Shi, J. Cao, Q. Zhang, Y. Li, and L. Xu, "Edge Computing: Vision and Challenges," IEEE Internet of Things Journal, vol. 3, no. 5, pp. 637646, Oct. 2016, doi: 10.1109/JIOT.2016.2579198.

[9] M. Tran, "Performance evaluations of the new GPS L5 and L2 Civil (L2C) signals," Navigation, vol. 51, no. 3, pp. 199-212, 2004.

[10] H. Wong, K.-M. Luk, C. H. Chan, Q. Xue, K. K. So, and H. W. Lai, "Small antennas in wireless communications," Proceedings of the IEEE, vol. 100, no. 7, pp. 2109-2121, 2012.

[11] H.-M. Chen, J.-M. Chen, P.-S. Cheng, and Y.-F. Lin, "Feed for dual-band printed dipole antenna," Electronics Letters, vol. 40, no. 21, pp. 13201322, 2004.

[12] M. Abu, M. K. A. Rahim, M. K. Suaidi, I. M. Ibrahim, and N. M. Nor, "A meandered triple-band printed dipole antenna for RFID," in 2009 Asia Pacific Microwave Conference, Dec. 2009, pp. 1958-1961, doi: 10.1109/APMC.2009.5385292.

[13] S.-Y. Chen, Y.-C. Chen, and P. Hsu, "CPW-fed aperture-coupled slot dipole antenna for tri-band operation," IEEE Antennas and Wireless Propagation Letters, vol. 7, pp. 535-537, 2008.

[14] S. U. Rahman, M. I. Khan, N. Akhtar, and F. Murad, "Planar dipole antenna for tri-band PCS and WLAN communications," in 2016 Progress in Electromagnetic Research Symposium (PIERS), 2016, pp. 4397-4400.

[15] K. Chang, H. Kim, and Y. J. Yoon, "A triple-band printed dipole antenna using parasitic elements," Microwave and optical technology letters, vol. 47 , no. 3, pp. 221-223, 2005.

[16] J. M. Floc'h and I. B. Trad, "Design of multiband and wideband antennas with printed dipole," in 2015 IEEE 15th Mediterranean Microwave Symposium (MMS), Nov. 2015, pp. 1-4, doi: 10.1109/MMS.2015.7375483.

[17] Y.-H. Suh and K. Chang, "Low cost microstrip-fed dual frequency printed dipole antenna for wireless communications," Electronics Letters, vol. 36, no. 14, pp. 1177-1179, 2000.

[18] Chih-Ming Su, Hong-Twu Chen, and Kin-Lu Wong, "Printed dual-band dipole antenna with U-slotted arms for 2.4/5.2 GHz WLAN operation," Electronics Letters, vol. 38, no. 22, pp. 1308-1309, Oct. 2002, doi: 10.1049/el:20020919.

[19] S. Jeon, Y. Yu, and J. Choi, "Dual-band slot-coupled dipole antenna for $900 \mathrm{MHz}$ and $2.45 \mathrm{GHz}$ RFID tag application," Electronics letters, vol. 42, no. 22, pp. 1259-1260, 2006.

[20] T.-L. Chen, "Multi-band printed sleeve dipole antenna," Electronics Letters, vol. 39, no. 1, pp. 14-15, 2003.

[21] K. Jebbawi, M. Egels, and P. Pannier, "Triple-band printed dipole antenna for RFID/GPS/BLE applications," Progress In Electromagnetics Research M, vol. 70, pp. 11-20, 2018.

[22] Y.-J. Chen, T.-W. Liu, and W.-H. Tu, "CPW-fed penta-band slot dipole antenna based on comb-like metal sheets," IEEE Antennas and Wireless Propagation Letters, vol. 16, pp. 202-205, 2016.

[23] R. J. Garbacz, "Modal expansions for resonance scattering phenomena," Proceedings of the IEEE, vol. 53, no. 8, pp. 856-864, 1965.

[24] R. Harrington and J. Mautz, "Theory of characteristic modes for conducting bodies," IEEE Transactions on Antennas and Propagation, vol. 19 , no. 5 , pp. $622-628,1971$

[25] "Feko - Altair HyperWorks." https://altairhyperworks.com/product/FEKO (accessed Jan. 15, 2020).

[26] A. Abbosh, "Accurate effective permittivity calculation of printed centerfed dipoles and its application to quasi Yagi-Uda antennas," IEEE Transactions on antennas and propagation, vol. 61, no. 4, pp. 22972300, 2012.

[27] Z. Živković, A. Šarolić, and V. Roje, "Effects of effective dielectric constant of printed short dipoles in electric field probes," in 10th International Symposium on Electromagnetic Compatibility, 2011, pp. $73-78$

[28] "Welcome to Python.org," Python.org. https://www.python.org/ (accessed Jan. 19, 2020). 
[29] A. D. Yaghjian and S. R. Best, "Impedance, bandwidth, and Q of antennas," IEEE Transactions on Antennas and Propagation, vol. 53, no. 4, pp. 1298-1324, 2005, doi: 10.1109/TAP.2005.844443. 